\title{
El análisis de la literatura biomédica en España en clave de diversidad cultural y de género
}

\author{
Ma LUISA JIMÉNEZ \\ Universidad de Sevilla \\ mljimenez@us.es \\ Ma Teresa Gijón \\ Universidad de Málaga \\ mtgijon@ugr.es \\ EMILIA MARTÍNEZ \\ Universidad de Granada \\ emilia.marmo@gmail.com
}

Recibido: 23.06 .2008

Aceptado: 26.05.2009

\section{INTRODUCCIÓN}

«Una práctica científica que omite cuestionarse a sí misma no sabe, en realidad, lo que está haciendo»

Pierre Bourdieu. Una duda radical.

La influencia de los valores en la práctica científica es una cuestión central para la sociología, que ha mostrado las limitaciones de considerar el conocimiento científico como verdad absoluta, objetiva, imparcial y exenta de prejuicios. La ciencia como hecho social está marcada por el contexto social e histórico en el que se desarrolla, siendo una representación consensuada y compartida que orienta la acción de los sujetos y con enormes implicaciones epistemológicas y sociales. Es la comunidad científica quien determina, en cada momento y área de trabajo, qué se considera conocimiento verdadero o falso, qué objetos son o no legítimos de estudio y la manera en que son analizados e interpretados. Desde un planteamiento sociológico, la definición dominante de la ciencia es especulativa, idealista, normativa y legitimadora y en consecuencia insatisfactoria en términos científicos (Iranzo y Blanco 1999). 
Retomando la necesidad expuesta por Bourdieu, es preciso que la ciencia se cuestione a sí misma para poder saber qué está haciendo y cómo lo está haciendo, planteándose cómo se preconstruyen, construyen y postconstruyen determinados objetos científicos, sus conceptos y nociones clasificatorias, sus instrumentos de observación y análisis y sus enfoques de acercamiento (Bourdieu 1995, 2002). La sociología ofrece herramientas conceptuales y metodológicas de reflexión y crítica para reconsiderar los procedimientos por los que determinados problemas científicos se configuran y se abordan, poniendo de manifiesto cómo los actores que intervienen en este proceso están socioculturalmente condicio$\operatorname{nados}^{1}$ y sus conocimientos socialmente situados (Haraway 1988, Harding 2003).

En este trabajo planteamos la metainvestigación como herramienta para la reflexión y autocrítica de los procesos de investigación científica, incorporando como marco de análisis e interpretación el estudio de la diferencia y la desigualdad desde un enfoque de diversidad cultural y de género. El concepto de metainvestigación, tal y como se emplea aquí, hace referencia a la relevancia y necesidad de analizar críticamente qué se investiga y, especialmente, cómo se investiga en los diferentes campos del saber, como estrategia fundamental de avance científico. Esta noción se sitúa en la línea reflexiva que se ha venido desarrollando en sociología desde las dos últimas décadas y que ha dado lugar a diversos enfoques basados en la metateorización [metatheory] (Ritzer 1991, Zhao 1991) y en la metametodología (Noblit y Hare 1988) como estrategias de síntesis y análisis y en definitiva de progreso científico. La metateorización conlleva el estudio sistemático de la teoría sociológica entendida en tres sentidos principales: como medio para un mejor y más profundo entendimiento de la teoría existente, como preludio de una teoría posterior y como fuente de perspectivas teóricas integradoras (Ritzer 1991). Otros enfoques se ubican más en el terreno de los métodos y los resultados de la investigación como la metasíntesis [metasynthesis]. Se trata de un tipo de investigación donde las fuentes de datos son las investigaciones precedentes; pero es algo más que la acumulación de los resultados de diversos estudios ya que supone un análisis crítico e interpretativo profundo del estado de la teoría y de la metodología que afecta a una determinada área de investigación, seguido de una síntesis e integración (Thorne et al 2004, Zimmer 2006, Bondas y Hall 2007). Entre los diversos abordajes metasintéticos $^{2}$, destacamos la propuesta de Lela Zimmer (2006) relativa a la inda-

\footnotetext{
${ }^{1}$ La incorporación de los valores en la práctica investigadora es inevitable, lo mismo que la experiencia personal, social y científica de quien investiga. El seguimiento del método no garantiza la ausencia de valores, por lo que la única forma de controlar su papel es teniéndolos presentes, incluyéndolos conscientemente en la investigación. Por tanto se trataría de un conocimiento parcial, localizado y crítico frente a las aspiraciones universales y holísticas características del modo hegemónico de hacer ciencia (Ortiz, 2002:30).

2 Dentro de la metasíntesis se han identificado diferentes modalidades: el metaestudio [metastudy], tratando de diferenciar entre análisis y síntesis; el metamétodo [metamethod], que examina las características metodológicas para determinar la manera en la que los métodos, frecuentemen-
}

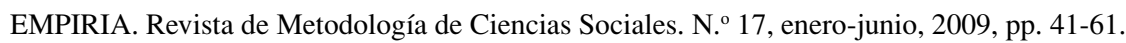
ISSN: $1139-5737$ 
gación cualitativa sobre los resultados de investigaciones cualitativas en relación a un tema concreto. El objetivo es la elaboración, a través del diálogo con los textos, de una interpretación de las interpretaciones de los datos primarios realizadas por sus autores o autoras. La metasíntesis cualitativa -a diferencia de la cuantitativa que busca una reducción de los datos- persigue generar nuevos significados e interpretaciones (Zimmer 2006). Con la metainvestigación también se pretende establecer un diálogo con los textos, pero con la especificidad de que los datos objeto de análisis están conformados por el discurso científico volcado en los artículos o en otras plataformas de transmisión del conocimiento científico, buscando interpretar cómo se definen y cómo se interpretan los problemas de investigación y sus resultados desde un área concreta de investigación.

En este estudio hemos centrado nuestro interés en el campo de la biomedicina y, de manera más particular, en cómo se investiga desde este paradigma el proceso de salud-enfermedad-atención de personas migrantes de origen extranjero $^{3}$. Nos hemos centrado en la biomedicina, porque nos parecía un reto aplicar esta duda radical (Bourdieu 1995) a un campo del saber donde los valores de objetividad, neutralidad, universalidad e independencia se establecen como los principios rectores de su modo de funcionamiento, organización y legitimación ${ }^{4}$. Desde estas premisas se tiende a esencializar, naturalizar y/o reificar relaciones de género e interculturales, despojándolas de su contenido social. Por otra parte, la migración extranjera constituye un campo de reflexión especialmente sensible en relación a la diversidad y la desigualdad social, y más aún teniendo presente que en la actualidad las relaciones interétnicas en los nuevos escenarios migratorios se configuran como problema social y sanitario de gran relevancia y proyección mediática y política.

Hemos optado como estrategia de acercamiento a esta cuestión el análisis de la literatura científica, ya que entendemos los textos de las publicaciones como

te cualitativos, son interpretados y cómo afectan al significado de los resultados de la investigación; o el metaanálisis [metaanalysis], como estrategia de investigación de las publicaciones mediante revisiones comprehensivas de la literatura (Bondas y Hall 2007).

${ }^{3}$ La delimitación conceptual de este término es compleja, ya que a nivel internacional no hay una definición universalmente aceptada. Se suele aplicar a las personas y a sus familiares que toman libremente la decisión de emigrar y sin intervención de factores externos y que van a otro país o región con miras a mejorar sus condiciones sociales y materiales y sus perspectivas y las de sus familias (OIM, 2006).

${ }^{4}$ De acuerdo con Eduardo Menéndez el modelo médico hegemónico se refiere al «conjunto de prácticas, saberes y teorías generadas por el desarrollo de lo que se conoce como medicina científica, que permite entender la racionalidad bio-médica y el funcionamiento de la ideología dominante y técnica del saber médico» (Menéndez 1990:84 y 1996:10). Este campo se ha identificado en Occidente como la única forma legítima de atender las enfermedades y se caracteriza por su «biologismo, a-sociabilidad, a-historicidad, aculturalismo, individualismo, eficacia pragmática, orientación curativa, relación médico-paciente asimétrica y subordinada, exclusión del saber del paciente, profesionalización formalizada, identificación ideológica con la racionalidad científica, la salud/enfermedad como mercancía, tendencia a la medicalización de los problemas, tendencia a la escisión entre teoría y práctica» (Menéndez 2003:194).

EMPIRIA. Revista de Metodología de Ciencias Sociales. N. ${ }^{\circ}$ 17, enero-junio, 2009, pp. 41-61. ISSN: 1139-5737 
medio de difusión del discurso y topos o lugar de (re)presentación de significaciones y prácticas investigadoras. No buscamos realizar una síntesis o integración de los resultados producidos en este campo, como pretendería una revisión bibliográfica al uso, sino que intentamos ir más allá: analizar y desentrañar, además de cuáles son los problemas o cuestiones científicamente relevantes, cómo son construidos y abordados. De manera específica, nos interesa indagar la presencia o ausencia de sesgos etnocéntricos y androcéntricos que puedan afectar a este proceso.

\section{CUESTIONANDO EL MODO HEGEMÓNICO DE HACER CIENCIA}

El modo hegemónico occidental de hacer ciencia ha sido discutido desde diversas posiciones. Aquí nos centramos en dos posiciones de crítica ${ }^{5}$. Por una parte, las teorías feministas han puesto al descubierto su carácter marcadamente androcéntrico ${ }^{6}$ en la creación y definición de los problemas de investigación y en cómo son afrontados. También, han criticado la omisión e invisibilidad de las mujeres como sujetos y objetos legítimos del quehacer científico (Keller 1982, Harding 1991, Oakley 1998, Moore 1999, Miqueo 2001, Ortiz 2006). Por otra parte, se ha denunciado su carácter etnocéntrico (Nencel y Pels 1991), que se manifiesta cuando el investigador o investigadora ve «a 'los otros', pueblos o grupos sociales, desde los parámetros hegemónicos vigentes en la sociedad del investigador, a la que considera, implícita o explícitamente, superior, y en esa medida, observa la realidad desde ideas preconcebidas y conceptos acuñados en su mundo social» (Mozo y Tena 2003:11). En definitiva, percibe la diversidad desde enfoques alejados de la pluralidad de manifestaciones culturales. Sobre esta cuestión, Sandra Harding ha destacado cómo «la estructura social de la ciencia, muchas de sus aplicaciones y tecnologías, sus formas de definir los problemas de investigación, y de diseñar experimentos, sus modos de construir y conferir significados son no sólo sexistas, sino también racistas, clasistas y coercitivos en el plano cultural» (Harding 1996:11).

Además del etnocentrismo y androcentrismo es preciso considerar la existencia de otras formas de jerarquía que producen un acercamiento sesgado al

\footnotetext{
${ }^{5}$ Otras posiciones que han examinado de modo crítico la ciencia moderna se han situado en el contexto de las luchas contra el racismo, el colonialismo, el capitalismo y la homofobia, realizando análisis sobre los usos y abusos de la ciencia a favor de los grupos dominantes (Harding 2003).

${ }^{6}$ «El androcentrismo se refiere a las formas de conocimiento y supone utilizar en cualquier investigación o análisis una perspectiva que surge de la experiencia social masculina, especialmente de la experiencia social de varones occidentales, blancos y de clase media. El androcentrismo implica la identificación de lo masculino con lo humano en general; y a su vez, la equiparación de lo humano en general con lo masculino, lo que lleva a constituir lo masculino como la norma. El androcentrismo en el método científico y su aplicación y ha estado y está presente en todas las ramas de la ciencia» (Ortiz 2006:42).
}

EMPIRIA. Revista de Metodología de Ciencias Sociales. N. ${ }^{\circ}$ 17, enero-junio, 2009, pp. 41-61. ISSN: $1139-5737$ 
análisis de la realidad social. Éstas se estructuran en torno a una amplitud de categorías de desigualdad social como género, cultura, etnicidad, (dis)capacidad, clase social, edad, religión, orientación sexual o localización geográfica, entre otras (Burke y Eichler 2006). También es necesario prestar atención al modo en que las estructuras jerárquicas interactúan, se retroalimentan e influyen en los procesos de generación, legitimación y difusión de los conocimientos científicos como verdades absolutas a lo largo de la historia. En el tema que nos ocupa, supone «plantear que el etnocentrismo adquiere distinto significado según se aplique a uno u otro sexo» (Mozo y Tena 2003:16). Del mismo modo, el androcentrismo afecta de diferente manera a las mujeres en función de su pertenencia -o no- a determinadas categorías sociales. Todo ello, teniendo en cuenta que la forma en que se presenta esta interacción de alteridades depende del contexto y el momento en el que se realizan las clasificaciones o distinciones (Nash 2001). En este sentido «es posible detectar, o descartar, el componente sexista [y, añadimos nosotras, el componente etnicista] de este sistema de codificación de la realidad que llamamos ciencia [...]. También es posible analizar el modo concreto en que se introyectan esos valores culturales propios de esa comunidad científica, incluidos los estereotipos sexuales [y etnocéntricos], en la elección de problemas que hay que investigar, en la elección de objetos de estudio de investigación (sujetos de estudio o material empírico experimental), en la elección de los hechos relevantes que constituyen los datos científicos o evidencias, o en la elección de las palabras -sustantivos, adjetivos y verbos- con que se describen los hechos observados (datos de los resultados), se explican los datos (teorías) o se comunica ese conocimiento dentro de la comunidad de expertos o fuera de ella (docencia o divulgación)» (Miqueo 2001:101).

Los textos de las publicaciones operan como fotogramas reveladores de los objetos y sujetos investigados y de su construcción epistemológica ${ }^{7}$. Concebimos, el análisis crítico de la literatura como una potente herramienta de metainvestigación por tres motivos fundamentales. Primero, porque los trabajos publicados en revistas científicas constituyen unos productos directos del proceso de investigación y con un gran impacto académico y social. Segundo, porque suponen un soporte de epistemas normativos donde se plasman en forma de discurso las teorías, ideologías y prácticas que guían y condicionan la investigación, sus agendas y metodologías; del mismo modo que vehiculan la definición y reificación de diferencias que pueden ocultar relaciones de desigualdad. Un tercer motivo responde estrictamente a criterios pragmáticos de disponibilidad, accesibilidad de la información y eficacia en la recogida y análisis de los datos.

7 Además, son resultado de estrategias retóricas mediante las cuales las personas investigadoras fuerzan a las demás, a través de textos, archivos, documentos y artículos, a transformar en un hecho lo que en un principio era una opinión (Latour 1992:30). En este sentido, es preciso no perder de vista no sólo la influencia del macrocontexto social y cultural que rodea a las personas que investigan sino también las propias dinámicas y lógicas de acción y legitimación de la actividad investigadora frente al resto de la comunidad científica.

EMPIRIA. Revista de Metodología de Ciencias Sociales. N. ${ }^{\circ}$ 17, enero-junio, 2009, pp. 41-61. ISSN: $1139-5737$ 


\section{APUNTES METODOLÓGICOS}

Partiendo de estas premisas, mostramos, como ejemplo inicial y tentativo de metainvestigación, el procedimiento metodológico y los principales resultados de un estudio sobre las representaciones sociales, significaciones y prácticas en torno a las migraciones y la población migrante de origen extranjero en la literatura biomédica española. En primer lugar, se realizó una búsqueda bibliográfica sistemática de todos los artículos científicos sobre esta temática ${ }^{8}$ publicados, desde 1984 hasta 2006, e indexados en el Índice Médico Español, base gestionada por el Consejo Superior de Investigaciones Científicas. Optamos por delimitar nuestro análisis a esta base por ser el principal referente bibliográfico sanitario en España y, por tanto, una fuente esencial en la construcción del discurso biomédico. En este proceso encontramos una serie de dificultades. Primero, porque la cantidad de estudios biomédicos sobre «inmigración» publicados en los últimos años en España, su calidad y su temática de estudio difieren sustancialmente. Otro problema añadido se refiere a la búsqueda y localización final de los títulos elegidos, ya que no siempre se hallaban accesibles en las bases de datos consultadas o bien estaban vivos en catálogo, pero no disponibles electrónica o manualmente. Todo ello, implica que dificultosamente se pueda establecer una lista completa y exhaustiva. No obstante teniendo en cuenta estas limitaciones y tras aplicar unos criterios estratégicos de selección y exclusión ${ }^{9}$, el corpus documental definitivo ascendió a un total de 113 artículos originales y de revisión. Estos artículos y la información que contenían fueron organizados en una base datos de carácter cualitativo y cuantitativo ${ }^{10}$.

En la fase de análisis, diseñamos una herramienta para mirar e interpretar la producción científica desde perspectivas más sensibles a la desigualdad de género y a la diversidad cultural, para así detectar o descartar posibles sesgos de aproximación ${ }^{11}$. Desde esta posición, adoptamos el concepto de instrumento óptico de Donna Haraway en el que se pueden combinar diferentes lentes «que tienen el propósito de producir no tanto efectos de distanciamiento, como efec-

${ }^{8}$ Se tomaron como términos de búsqueda para la recuperación de artículos científicos sobre «inmigración» los siguientes: inmigración, inmigrante/s, migración, migrante/s, emigrante/s, extranjero/s, extranjera/s, minoría, etnia/s, étnico/s, étnica/s y etnicidad. Estos términos se aplicaron a los campos del título, resumen y descriptores o palabras clave.

9 Se descartaron notas clínicas, editoriales, cartas al director, comunicaciones y ponencias a congresos, así como los documentos cuyo texto completo no estaba disponible ni accesible después de una intensiva labor de localización.

10 Se analizó el texto completo de todos los artículos, por una parte codificando y cuantificando temáticas y cuestiones relevantes a los objetivos de este trabajo, y por otra, seleccionando fragmentos de discurso significativos para su posterior interpretación.

11 Contamos con unos pocos antecedentes empíricos como son los estudios de Eichler (2001) en el ámbito europeo y del equipo de Cerón-Míreles (2006) en el contexto latinoamericano. En estos trabajos se evidencia, desde un abordaje analítico del contenido de los artículos biomédicos, la ausencia de un enfoque de género y su impacto negativo en los criterios de validez y de calidad científicos.

EMPIRIA. Revista de Metodología de Ciencias Sociales. N. ${ }^{\circ}$ 17, enero-junio, 2009, pp. 41-61. ISSN: 1139-5737 
tos de conexión, de encarnación y de responsabilidad con algún otro lugar imaginado que ya podemos aprender a ver y a construir» (Haraway 1999:122). Este instrumento óptico intercala dos filtros ${ }^{12}$ de identificación y evaluación de sesgos ${ }^{13}$ de corte etnocéntrico/etnicista y androcéntrico/sexista. Cada filtro está compuesto por una serie de cuestiones de detección que permiten concretar el grado de (in)sensibilidad de género o hacia la diversidad cultural y étnica de la literatura científica. Estas cuestiones nos sirvieron para interrogar a cada uno de los artículos seleccionados. Las respuestas que nos daban estos trabajos fueron registradas tanto a nivel cuantitativo como cualitativo.

El primero de los filtros se configura desde un enfoque sensible a la diversidad cultural. Éste se inscribe, siguiendo a Mary Nash, «en los discursos de alteridad, de definición del otro/a, en la formación de subjetividades individuales y colectivas o en su expresión como identidades [...]. Se interesa por las definiciones abiertas donde se constituyen, se desmarcan o desaparecen las diferencias y también por descifrar los discursos, representaciones culturales y prácticas sociales que delinean la visión del otro/a y su reconstitución a través del reflejo de esta mirada» (Nash 2001: 29). A través de este filtro, pretendemos analizar a partir del discurso biomédico académico cómo, aquí y ahora, se construyen las temáticas y objetos de estudio de la salud de las y los inmigrantes extranjeros y cómo se investigan y puntualizan las enfermedades o malestares y se aborda el proceso de atención sanitaria en esta población. Para ello, aplicamos a los artículos unas cuestiones específicas de detección de sesgos etnocéntricos y etnicistas que se indican en el cuadro 1.

Cuadro 1. Cuestiones de detectación de sesgos etnocéntricos y etnicistas en la literatura científica.

1. ¿Cuáles son las cuestiones de investigación relevantes en la población inmigrante extranjera?

2. ¿Se atiende a la diversidad de la población inmigrante extranjera?

3. ¿Se tiene en cuenta la dimensión cultural en el planteamiento de los problemas de investigación y en la interpretación de los resultados?

4. ¿Se reproducen y transmiten estereotipos etnocéntricos y etnicistas sobre la población inmigrante extranjera y su relación con el proceso salud-enfermedadatención?

12 Aunque podrían estar presentes otros filtros basados en una perspectiva de análisis desde las clases sociales, la orientación sexual o la relación con la capacidad, por ejemplo (ver, a este respecto, el trabajo de Burke y Eichler 2006).

${ }^{13}$ Los sesgos son errores y limitaciones, que consciente o inconscientemente, se producen de manera sistemática y desvirtúan el proceso de investigación, poniendo en grave riesgo su validez y rigor, ya que se aproximan a la realidad y dan cuenta de ella de un modo parcial y equívoco.

EMPIRIA. Revista de Metodología de Ciencias Sociales. N. ${ }^{\circ}$ 17, enero-junio, 2009, pp. 41-61. ISSN: 1139-5737 
El segundo filtro parte de un enfoque sensible al género, que busca fundamentalmente sacar a la luz las desigualdades entre mujeres y hombres y explicarlas desde el contexto histórico, social, cultural y económico donde se desarrollan, reproducen y legitiman (Tubert 2003, Maqueira 2005). En relación con el filtro anterior, se trata de profundizar y desvelar la experiencia colectiva de mujeres y hombres inmigrados extranjeros y cómo se presentan y se sostienen desde la mirada biomédica las desigualdades entre los sexos y los estereotipos asociados por razón étnica y sexual. Desde estas premisas tratamos de identificar qué tipo de problemas de investigación son definidos como significativos en mujeres y en varones inmigrantes extranjeros y las diferencias en su construcción como femeninos o masculinos. A continuación, valoramos cómo los estudios contemplan las diferencias entre los sexos -si las contemplan-, primero explorando si éstos atienden al sexo como variable ${ }^{14}$; y segundo si incorporan el género como categoría o dimensión de análisis a la hora de investigar las problemáticas en salud. Así también, pretendemos evaluar el posicionamiento de los discursos plasmados en los textos frente a las desigualdades étnicas y de género y su papel en la reproducción y transmisión -o no- de estereotipos sexistas o/y androcéntricos. A este respecto, también indagamos cómo estereotipos sexistas interactúan y se recomponen mediatizados por estereotipos de corte etnocéntricos. Las cuestiones específicas de detección se recogen en el cuadro 2.

\section{Cuadro 2. Cuestiones de detectación de sesgos androcéntricos} y sexistas en la literatura científica.

1. ¿Cuáles son las cuestiones de investigación relevantes en la población inmigrante extranjera femenina y masculina?

2. ¿Se considera el sexo como variable de estudio y de análisis?

3. ¿Se tiene en cuenta la dimensión de género en el planteamiento de los problemas de investigación y/o en la interpretación de los resultados?

4. ¿Se reproducen y transmiten estereotipos androcéntricos y sexistas sobre las relaciones de mujeres y hombres con el proceso salud-enfermedad-atención?

Ambos enfoques de observación y análisis se encuentran interrelacionados y deben ser planteados de forma interseccional ${ }^{15}$. En este sentido, es preciso subrayar «el importante papel que debe desempeñar el reconocimiento de las di-

${ }^{14}$ La desagregación de los datos por sexo constituye un requisito fundamental y necesario, aunque en ningún caso único ni suficiente, para comenzar a tratar las diferencias y las semejanzas entre los sexos.

15 El concepto de interseccionalidad [intersectionality] emergió como respuesta a las críticas a los estudios de género por su tendencia esencializadora de la experiencia de las mujeres, configurándose como un nuevo instrumento analítico de «las relaciones entre las múltiples dimensiones y modalidades de relaciones sociales y formaciones subjetivas» (McCall 2005: 1771).

EMPIRIA. Revista de Metodología de Ciencias Sociales. N. ${ }^{\circ}$ 17, enero-junio, 2009, pp. 41-61. ISSN: 1139-5737 
ferencias de género dentro de los grupos raciales y culturales y de las diferencias culturales dentro de los grupos de género en las epistemologías y políticas emancipatorias» (Harding 1996:18).

\section{LA SALUD DE LA POBLACIÓN INMIGRANTE COMO «OBJETO CIENTÍFICO»}

Desde el prisma de la diversidad cultural, encontramos en gran parte de la literatura analizada la presencia de visiones esencialistas, estereotípicas y etnocéntricas sobre la salud de las personas migrantes de origen extranjero y su relación con el sistema biomédico de atención sanitaria. El primer mecanismo reduccionista está ligado a la determinación de los sujetos de investigación clasificables como «inmigrantes» como aquellas personas provenientes de países «pobres», «en desarrollo» o del «Tercer Mundo»y, la mayoría de las veces, en situación de «ilegalidad» 0 «sin papeles». Se obvian otras realidades migratorias de origen comunitario, que paradójicamente conforman casi la mitad de la población extranjera residente en territorio español y que por su perfil sociodemográfico - una parte considerable son personas mayores que se encuentran jubiladas y habitan en zonas costeras- implican un elevado coste sanitario (Rodríguez et al. 2005, Solé 2006).

En el cuadro 3, mostramos un resumen de las respuestas obtenidas tras la aplicación de la herramienta de detección de sesgos etnocéntricos. Respecto a la primera cuestión de análisis, se observa una etnificación de ciertos problemas de salud al estudiar dolencias y enfermedades -que también pueden afectar a la población en general- de forma más prevalente y específica en la población inmigrante. Muchas de estas enfermedades se delimitan en función de su vinculación a su procedencia geográfica de países en vías de desarrollo y, en definitiva, de su distancia social y cultural. Las que se asocian con mayor frecuencia a la población inmigrante son las infecciosas transmisibles (especialmente, tuberculosis, VIH-SIDA y hepatitis) implicando graves reduccionismos en la construcción sanitaria y social de la salud de las mujeres y varones migrantes extranjeros. Además, se da un sobredimensionamiento de los aspectos relacionados con la salud mental -en particular, con el denominado «duelo migratorio»-, a pesar de que en la literatura internacional existe controversia sobre esta cuestión. También cabe reseñar la creciente importancia de las temáticas de investigación relacionadas con un mejor conocimiento de las demandas asistenciales de la población inmigrante y su perfil sociosanitario y también con cuestiones vinculadas a la atención sanitaria. Si bien en este último aspecto, convergen visiones diferentes que oscilan desde la conceptualización de estos nuevos grupos usuarios como un reto o desafío asistencial y formativo hasta su interpretación como una amenaza o nueva carga sanitaria y económica. 
Cuadro 3. Presencia de sesgos etnocéntricos y etnicistas en los artículos biomédicos sobre migraciones y salud publicados en España. $N=113$.

\begin{tabular}{|c|c|}
\hline Temas de investigación relevantes & $\begin{array}{l}\text { - Enfermedades infecciosas: } 28,3 \% \\
\text { - Atención sanitaria: } 18,6 \% \\
\text { - Motivos de consulta: } 17,7 \% \\
\text { - Enfermedades mentales: } 10,6 \% \\
\text { - Embarazo-Parto-Reproducción: } 8 \% \\
\text { - Alimentación-Nutrición: 5,3\% } \\
\text { - Vacunación: } 3,5 \%\end{array}$ \\
\hline $\begin{array}{l}\text { No se atiende a la diversidad de la } \\
\text { población inmigrante extranjera }\end{array}$ & $72,6 \%$ \\
\hline $\begin{array}{l}\text { No se tiene en cuenta la dimensión } \\
\text { cultural en el planteamiento de los } \\
\text { problemas de investigación y en la } \\
\text { interpretación de los resultados }\end{array}$ & $60,2 \%$ \\
\hline $\begin{array}{l}\text { Reproducción de estereotipos } \\
\text { etnocéntricos y etnicistas }\end{array}$ & $\begin{array}{l}\text { - Población inmigrante extranjera } \\
\text { limitada al «Tercer Mundo»- } \\
\text { «Ilegalidad» } \\
\text { - Coste económico - Carga asistencial } \\
\text { - Riesgo de contaminación - Enferme- } \\
\text { dades importadas - Grupo de riesgo } \\
\text { epidemiológico } \\
\text { - Conflictividad cultural y social / } \\
\text { Grupos inadaptados }\end{array}$ \\
\hline
\end{tabular}

Respecto a la segunda cuestión analizada, se advierte, en general, una homogeneización de la población inmigrante en base a una contemplación de características o rasgos diferenciables entre la «población autóctona» y la «población inmigrante», que puede ser consecuencia de la búsqueda de mecanismos que simplifiquen la variedad y diversidad de realidades sociales y sanitarias ${ }^{16}$. En este caso, es la variable «procedencia geográfica» la delimitadora de las diferencias entre grupos, empleada desde una posición marcadamente etnocéntrica, sin contemplar en ningún momento la autoadscripción étnica o cultural de las personas o grupos de los que hablan. La «procedencia geográfica»-casi siempre identificada con la nacionalidad o zonas geográficas más o menos amplias y coincidentes con los países de rentas más bajas- aparece como categoría supuestamente natural y objetivable, sin toda la problemática que pueden arrastrar

16 En este sentido, cabe señalar que «la construcción de la diferencia no es más que una nueva forma de presentar las distancias culturales, sociales y políticas que son legitimadas bajo la apariencia de ausencia de jerarquías sociales pero que ocultan un refinado mecanismo de exclusión» (García Castaño, Granados y Pulido 1999:2). 
otros términos como «raza» o «etnia». Se produce una geografización de la diferencia cultural con resultados simplificadores y esencializadores en virtud de fronteras casi siempre difusas (Gijón, Jiménez Rodrigo y Martínez Morante 2006). La inclusión de la variable «procedencia extranjera» como categoría de estudio en las investigaciones biomédicas ha sido y es objeto de debate y polémica $^{17}$. De hecho, la forma en que es definida esta categoría de análisis -de manera similar al tratamiento de la variable «etnia» (Senior y Bhopal 1994, Kaplan y Bennett 2003) - y sus valores asociados, puede entrañar en la práctica una amplificación, e incluso invención, de diferencias sociales y culturales, con el riesgo que esto supone de legitimación científica de la desigualdad. Esto va aparejado a la insensibilidad hacia la pluralidad de experiencias personales y grupales en salud y la influencia de las especificidades culturales en el modo de experimentar, vivir e interpretar la salud y la enfermedad. Alrededor de siete de cada diez estudios analizados no atienden a las particularidades étnicas, sociales o económicas al margen o dentro de la procedencia geográfica «extranjera»; variable que, a menudo, es construida sobre criterios cuestionables, no sólo por su desconexión de lo social sino también por la falta de sistematicidad, exhaustividad o exclusividad de sus categorías de clasificación ${ }^{18}$. En relación a la contemplación de la dimensión cultural tanto en el planteamiento de los temas de investigación como en sus resultados, sólo cuatro de cada diez artículos se refieren a aspectos «culturales». Si bien, en la mayor parte de los casos la «cultura» es entendida como un cajón de sastre donde se incluye todo lo diferente e incomprensible en torno a creencias y comportamientos en salud o estilos de vida y también como una barrera para la adaptación de las personas que migran. Incluimos el siguiente fragmento de discurso como ilustración:

«Todos estos niños tienen un origen muy distinto, pertenecen a distintos continentes, razas, culturas, religiones, etc. [...] Se comprende que querer agrupar las enfermedades más frecuentes de estos niños puede ser algo artificioso, debido a los orígenes tan distintos y a que se trata de individuos únicos. [...] Todos los niños inmigrantes tienen algo en común. Sus padres han emigrado tratando de dejar atrás unas circunstancias de desfavorecimiento económico, que no

17 Esta creciente productividad de artículos biomédicos sobre población inmigrante extranjera en España se explica, entre otros elementos, por la consolidación de la «etnicidad» y/o la «procedencia geográfica» como variables de diferenciación y comparación entre grupos humanos -al estilo de otras como la edad, el sexo o el hábitat de residencia- de gran relevancia para los estudios epidemiológicos en su pretensión de estudiar la distribución, frecuencia, determinantes, relaciones y predicciones de los factores relacionados con la salud y la enfermedad. De hecho, estas nuevas variables implican -dentro de las lógicas científicas biomédicas- una ampliación del campo de estudio. El riesgo aparece cuándo estas variables epidemiológicas se definen y se usan sin conexión con lo social sin cuestionar su relevancia ni su necesidad.

${ }^{18}$ Incluimos algunas de las clasificaciones de la población inmigrante utilizadas en los estudios analizados: «gitanos», «negros», «orientales», «árabes» y «blancos» (ID 9); «Sudamérica», «Centroamérica», «África» y «Filipinas» (ID 18); «Iberoamérica», «Magreb», «Europa del Este», «Asia», «África», «UE» y «Norteamérica» (ID 76); «África», «Centroamérica-Caribe», «Sudamérica» $\mathrm{y}$ «Asia» (ID 90).

EMPIRIA. Revista de Metodología de Ciencias Sociales. N.o 17, enero-junio, 2009, pp. 41-61. ISSN: $1139-5737$ 
siempre termina al llegar al nuevo país. A todos les toca vivir en una cultura distinta de la suya (ID 24).

«La primera barrera entre ellos y la población nativa es el lenguaje, no comprenden qué es lo que ocurre a su alrededor, tienen problemas con la aceptación de diferentes alimentos, clima y estilo de vida, con otra cultura y sistemas de valores. Tienen inseguridad sobre su futuro, además la actitud de la población autóctona frente a ellos hace sus vidas muy duras e incluso intolerables [...]. Si los trastornos psiquiátricos se consideran como el resultado de los efectos psicosociales del proceso de emigración, esto podría ser debido al gradual estrés de aculturación» (ID 8).

En otros artículos, también se transmite la idea de que es a través del contacto, el reconocimiento y el intercambio cultural la vía para la integración y una mejor atención sanitaria:

«La inmigración es un hecho positivo enriquecedor para el país que la acoge, tanto cultural como económica y demográficamente. [...] El conocimiento de cada realidad y cada comunidad tiene sus peculiaridades, permite la programación de unas actividades en función de unos objetivos que sólo salen de un estudio previo. La atención integral a este grupo emergente de población favorece no sólo el intercambio cultural, sino que evita la «guetización» y por ende los problemas sociosanitarios que derivan de la marginación y la pobreza» (ID 21).

Igualmente, tampoco suele atenderse a los condicionantes de los procesos migratorios ni a los contextos sociales y sistemas sanitarios de sus lugares de origen. Parece que las vidas de las mujeres y varones migrantes comienzan en el momento de migrar y que permanecen en el tiempo siendo inmigrantes, sin considerar, entre otros aspectos, el período de residencia, los procesos de cambio y enculturación o el grado de estructuración de sus redes sociales o su capital social. Otro aspecto que contribuye a la acentuación de las diferencias culturales -vinculadas a las geográficas- es la consideración de los problemas de salud de la población inmigrante en relación a su impacto en la salud de la mayoritaria. Como se observa repetidamente en el caso de las enfermedades infecciosas y transmisibles, también denominadas en algunos artículos como «importadas»:

«La incorporación de personas procedentes del llamado Tercer Mundo no sólo supone un impacto económico en la gestión de los recursos dedicados al sector de la salud, sino que supone la aparición de nuevas enfermedades, o antiguas ya erradicadas, y la incorporación en la población extranjera de las propias de nuestra civilización y costumbres» (ID 51).

«El aumento de nuevos casos de tuberculosis procedentes de países en vías de desarrollo ha contribuido a desequilibrar la tendencia decreciente de la enfermedad que venía observándose en los últimos años» (ID 62).

Todos estos argumentos comportan conceptualizaciones de carga peyorativa y amenazadora para el «desarrollo» y el «bienestar autóctono» que pueden legitimar posiciones etnicistas. Sobre todo porque cuando una conceptualización emana y se instaura en el terreno de la ciencia tiende a su más sólida justificación (Gómez Rodríguez 2004). Un resultado de aproximaciones biomédicas 
sesgadas y descontextualizadas al fenómeno de la inmigración es la reproducción, transmisión y legitimación de estereotipos etnocéntricos y etnicistas. Los más frecuentes son aquéllos que circunscriben a la población migrante de origen extranjero al «Tercer Mundo», «países en desarrollo o subdesarrollados» y la perfilan como un grupo con dificultades de adaptación cultural y demandante de servicios sociales, económicos y sanitarias, que en algunos casos, son presentadas en términos de carga económica y de conflictividad social y cultural. En unos pocos artículos, se sigue presentando de modo más o menos implícito el riesgo de contaminación que puede comportar la inmigración. Estereotipos, que como bien apuntan otros estudios, deben ser desmontados mediante un mayor conocimiento, formación y «una mejor atención sanitaria de calidad, responsable y culturalmente coherente con esta nueva diversidad».

\section{LAS (IN)DIFERENCIAS ENTRE MUJERES Y VARONES INMIGRANTES EN EL DISCURSO BIOMÉDICO}

La visión etnicista y etnocéntrica de la salud de la población inmigrante extranjera está también afectada por distorsiones derivadas de concepciones androcéntricas y sexistas que condicionan diferencias y desigualdades en la representación del proceso salud-enfermedad-atención de mujeres y hombres en el discurso científico biomédico. Cómo se sintetiza en el cuadro 4, se observa una desatención recurrente hacia las diferencias sexuales y las desigualdades de género en torno a la salud de la población inmigrante extranjera.

Casi un cuarenta por ciento de los estudios analizados no considera las diferencias entre los sexos y prescinde de la variable sexo en el diseño de sus investigaciones y en el análisis de sus resultados. Cuando se tiene en cuenta, actúa en muchos casos como otra variable más, sin profundizar en las diferencias y desigualdades entre mujeres y varones respecto a las cuestiones estudiadas ni explorar su interacción con otras variables sociodemográficas y sanitarias. Por otra parte, hay grupos de edad donde se observa una asexualización de la salud y de la enfermedad, como ocurre en la infancia que aparece como la época de la vida menos sexuada. Los artículos hacen una referencia constante a «el niño inmigrante» sin prestar atención a las diferencias entre los sexos y mucho menos a su construcción sociocultural. Sin embargo, en las edades adultas es cuando se consideran las diferencias entre mujeres y varones, basadas la mayoría de las veces en criterios sexual-reproductivos.

La ignorancia del sexo no afecta de igual forma a mujeres y varones, ya que la tendencia general, cuando se prescinde del sexo como variable significativa, es que se estudien problemas y necesidades de salud que pueden afectar a ambos sexos -por ejemplo, enfermedades transmisibles, VIH-SIDA, demandas asistenciales o atención pediátrica- sólo o preferentemente en varones. Esto se plasma en dos tipos de prácticas fuertemente androcéntricas: identificar problemas de salud generales como problemas de salud de los hombres y, en el sentido

EMPIRIA. Revista de Metodología de Ciencias Sociales. N. ${ }^{\circ}$ 17, enero-junio, 2009, pp. 41-61. ISSN: 1139-5737 
Cuadro 4. Presencia de sesgos androcéntricos y sexistas en los artículos biomédicos sobre migraciones y salud publicados en España. IME, $N=113$.

\begin{tabular}{|c|c|c|}
\hline $\begin{array}{l}\text { Temas de investigación re- } \\
\text { levantes en torno a mujeres } \\
\text { y hombres }\end{array}$ & $\begin{array}{l}\text { Artículos centrados ex- } \\
\text { clusivamente en mujeres } \\
(\mathrm{n}=15 ; 13,27 \%) \\
\text { - Embarazo y parto (10) } \\
\text { - Consulta ginecológica } \\
\text { - }(2) \\
\text { Salud de hijos de ma- } \\
\text { dres inmigrantes (1) } \\
\text { - Enfermedades de } \\
\text { transmisión sexual - } \\
\text { Prostitución (1) } \\
\text { - Estado de salud de las } \\
\text { mujeres inmigrantes } \\
\text { (1) }\end{array}$ & $\begin{array}{l}\text { Artículos centrados ex- } \\
\text { clusivamente en hom- } \\
\text { bres (n=8; 7,08\%) } \\
\text { - Enfermedades infec- } \\
\text { ciosas (4) } \\
\text { - Estado de salud y nu- } \\
\text { trición infan- } \\
\text { til/adolescente (1) } \\
\text { - Circuncisión infantil } \\
\text { (1) } \\
\text { - Perforación duodenal } \\
\text { (1) }\end{array}$ \\
\hline $\begin{array}{l}\text { No se contempla el sexo } \\
\text { como variable de estudio y } \\
\text { análisis }\end{array}$ & \multicolumn{2}{|c|}{$38,9 \%$} \\
\hline $\begin{array}{l}\text { No se tiene en cuenta la di- } \\
\text { mensión de género en el } \\
\text { planteamiento de los pro- } \\
\text { blemas de investigación y/o } \\
\text { en la interpretación de los } \\
\text { resultados }\end{array}$ & \multicolumn{2}{|c|}{$84,1 \%$} \\
\hline $\begin{array}{l}\text { Reproducción de estereoti- } \\
\text { pos androcéntricos y sexis- } \\
\text { tas }\end{array}$ & \multicolumn{2}{|c|}{$\begin{array}{l}\text { - Esencialización de la salud de la mujer a lo se- } \\
\text { xual-reproductivo; reducción de las mujeres a la } \\
\text { edad fértil } \\
\text { - Proyecto migratorio dependiente del varón (mari- } \\
\text { do) } \\
\text { - Promiscuidad - Prostitución - Enfermedades de } \\
\text { transmisión sexual } \\
\text { - Vulnerabilidad emocional - Enfermedades menta- } \\
\text { les - Abuso de fármacos }\end{array}$} \\
\hline
\end{tabular}

contrario, extrapolar los resultados obtenidos sólo en varones a la población general, quedando las mujeres y sus especificidades excluidas e infraestimadas. Esto conlleva una falta de reconocimiento de los problemas de salud de las mujeres inmigrantes extranjeras. Cuando las investigaciones posicionan a las mujeres como objeto específico y diferenciado de estudio, se suele reducir su salud a la dimensión sexual-reproductiva, prevaleciendo enfoques mal orientados o parciales derivados de abordajes estereotipados y esencializadores hacia lo 
biológico de la experiencia de las mujeres. Esta práctica es también común en el tratamiento de la salud femenina en general (Rosser 1994, Bird y Rieker 1999, Im 2000, Inhorn y Whittle 2001). Las temáticas femeninas prevalentes en los artículos analizados se ocupan fundamentalmente del embarazo, parto y crianza y otros aspectos vinculados a las consultas ginecológicas; y de forma puntual, de las enfermedades de transmisión sexual relacionadas con el ejercicio de la prostitución, la salud de «los hijos» de madres inmigrantes y de la percepción subjetiva de su estado de salud. En los hombres, los problemas de salud estudiados son un poco más diversos y la mayoría están vinculados a enfermedades infecciosas y otras enfermedades consideradas como «adaptativas». Así también, cabe señalar que si en el caso de las mujeres la mayoría de los estudios se centra en la «edad fértil», son la niñez y la adolescencia las edades que centran una atención particular en los trabajos sobre los varones.

Se detecta, que en casi ocho de cada diez artículos revisados, una insensibilidad a los aspectos ligados al género, lo que implica no considerar las desigualdades entre mujeres y hombres inmigrantes extranjeros e ignorar la influencia de los sistemas normativos de género en las experiencias de salud y enfermedad. Estos sistemas están mediatizados por procesos de interacción, asimilación y resistencia entre los contextos de origen y de acogida. Cabe reseñar el riesgo de descontextualización al eludir las circunstancias sociales, económicas y culturales que envuelven los procesos migratorios de mujeres y varones y las relaciones de género. En esta línea, se promueve una esencialización de la mujer y el varón inmigrante, al no atender a otras desigualdades, por clase social, procedencia o generación, que operan junto a las étnicas.

Nos parece importante resaltar que una mirada reflexiva sobre la construcción biomédica de «la mujer inmigrante» deja traslucir la presencia de estereotipos sexistas y androcéntricos. Uno de los más persistentes es la tendencia a presentar los proyectos migratorios femeninos en torno a su dimensión reproductiva frente a la dimensión productiva de los proyectos migratorios masculinos y a valorarlos como dependientes y subordinados a los de los varones. Como muestra, incluimos el siguiente fragmento:

«[En referencia al perfil demográfico de la población estudiada] El grupo mayoritario corresponde a varones entre 20 y 30 años. Ello hace suponer que en el progreso de su estabilización tenderán a traer a sus mujeres y fundar familias en nuestro país» (ID 15).

Además, la sexualidad femenina extranjera se percibe como peligrosa y portadora de enfermedades. Lo que reproduce la idea de contaminación atribuida al colectivo inmigrante, pero que en el caso de las mujeres se torna aún más amenazadora al transmitirse a través de una sexualidad «no controlada» y desviada:

«Las mujeres inmigrantes, ante una penuria económica, pueden verse tentadas a la prostitución como un método de supervivencia y por tanto estar sometidas a un riesgo añadido de infección» (ID 31).

«Hemos querido valorar en nuestro trabajo aspectos sociales que rodean al nacimiento de hijos de madres inmigrantes, para considerar los factores que au- 
mentan el riesgo social, entre ellos, la alta incidencia de ausencia de pareja estable y la no afiliación a la Seguridad Social por malas condiciones laborales» (ID 112).

Otros estereotipos detectados, y que en gran medida se corresponden con estereotipos sexistas de alcance general, se refieren a la vulnerabilidad emocional de las mujeres, que las hace «más propensas» a padecer trastornos mentales o al abuso de fármacos.

La ignorancia de la dimensión de género y su influencia en el proceso de salud-enfermedad-atención supone una forma muy grave de error científico (Eichler 2001) con serias consecuencias sociales y sanitarias al contribuir a la perpetuación de las desigualdades existentes. Es preciso no olvidar las funciones y responsabilidades sociales de la ciencia en cuanto a la resolución de los problemas de las personas. Los vacíos de conocimiento generados por la persistencia y reproducción del etnocentrismo y el androcentrismo, plantean cuestiones sobre cómo los productos de la biomedicina se transfieren a la práctica sanitaria; lo cual afecta al entendimiento, diagnóstico, tratamiento y atención de los problemas y necesidades en salud.

\section{CONCLUSIONES}

«Todo pasa y todo queda, pero lo nuestro es pasar, pasar haciendo caminos...»

Antonio Machado. Proverbios y Cantares.

En este trabajo hemos querido proponer la necesidad de ir más allá en las revisiones de la literatura científica, indagando no sólo qué se investiga sino también cómo se investiga e identificando o descartando limitaciones y aspectos cuestionables; producto del posicionamiento social y epistemológico de la comunidad investigadora frente a poblaciones heterogéneas. En definitiva, trazando caminos que nos ayuden a comprender la forma en que determinados problemas y discursos científicos nacen y se construyen, conformando a su vez visiones del mundo con efectos sociales.

Aunque hemos tratado, por razones operativas y de orden expositivo, ambos conjuntos de sesgos por separado, creemos necesario incidir en cómo el etnocentrismo no afecta del mismo modo a mujeres y a hombres migrantes de origen extranjero. $\mathrm{Y}$ viceversa, el androcentrismo tampoco afecta de la misma manera a las mujeres inmigrantes extranjeras. A este respecto, observamos dos tendencias contradictorias y paradójicas que merecen mayor atención e indagación. Por una parte, se produce una visibilización de la diferenciación expresa de la población inmigrante de origen extranjero en virtud de unos criterios geográficos y/o étnicos indefinidos. Por otra parte, se percibe la invisibilización de las mujeres en la mayoría de los temas de investigación, con la excepción de los as- 
pectos vinculados a la reproducción y la maternidad, donde se hipervisualizan las experiencias femeninas.

Las limitaciones en el entendimiento de la interacción de factores sociales, culturales y económicos, y de los sistemas y relaciones de género que median en el proceso salud-enfermedad-atención ponen en cuestionamiento el grado de validez interna de sus resultados. Pero también, el de su validez externa, al no contemplar la diversidad de género dentro de los grupos étnicos ni la diversidad étnica dentro de los grupos en función del sexo. Uno de los principales riesgos afecta a los procesos de construcción de la diferencia y de la otredad en función de nuestros estándares culturalmente definidos y transmitidos (Gijón, Jiménez Rodrigo y Martínez Morante 2006). Las razones y explicaciones que subyacen en las significaciones y miradas de estos estudios nos aproximan al (re)conocimiento de desigualdades económicas, políticas y sociales, que encauzadas adecuadamente podrían separarse de la procedencia geográfica y relanzar el planteamiento y debate de la diversidad cultural en relación a la desigualdad étnica, de género o de clase. Como hemos mostrado, la metainvestigación puede funcionar como una herramienta de visibilización de aspectos no explícitos en las investigaciones biomédicas, capaz de resaltar la importancia de atender a la diversidad cultural y a la influencia del género en el proceso salud-enfermedad-atención, independientemente de que el lugar de procedencia de las personas investigadas remita a aquí o allí. Pues cualquier población, independientemente de su origen nacional, tiene códigos culturales diversos y propios, ante los cuales el sistema sanitario aún tiende a ser homogenizador, monocultural y etno-geo-socio-andro-céntrico. Los sesgos identificados, en general, manifiestan «problemas» propios del sistema sanitario sobre una población migrante ante la que médicamente se encuentra preparado, pero ante la que culturalmente aparecen liminalidades consecuentes a la rigidez organizacional de los servicios sanitarios frente a «nuevas» demandas y diversidades en salud (Comelles 2004, Comelles y Bernal, 2007). Estas limitaciones también pueden afectar a otros temas y problemáticas de investigación. Por lo que este esfuerzo también puede aplicarse a otras líneas de estudio y a otros aspectos del proceso de construcción del conocimiento y de la intervención social (Jiménez Rodrigo, Martínez Morante y García Calvente, 2008).

Siendo coherentes con esta propuesta metodológica de metainvestigación debemos reconocer y plantearnos cómo influyen nuestras posiciones sociales y epistemológicas en las que nos situamos como investigadoras. Estas posiciones nos marcan los criterios de valoración e interpretación de la producción científica, sin dejar de ser tan convencionales como los planteamientos pretendidamente objetivos y verdaderos que criticamos. Creemos que es precisa una crítica reflexiva que permita el avance hacia significaciones y prácticas investigadoras más conscientes y sensibles a la diversidad cultural y la desigualdad social y de género. Éste es un recorrido necesario para hacer mejor ciencia y promover que los hallazgos científicos se transfieran a la sociedad de forma más igualitaria y justa.

EMPIRIA. Revista de Metodología de Ciencias Sociales. N. ${ }^{\circ}$ 17, enero-junio, 2009, pp. 41-61. ISSN: 1139-5737 


\section{AGRADECIMIENTOS}

Queremos agradecer a Eduardo Bericat y Manolo Río sus enriquecedoras sugerencias y matizaciones a este trabajo.

\section{BIBLIOGRAFÍA}

BIRD, Chloe \& RIEKER, Patricia (1999): «Gender matters: an integrated model for understanding men's and women's health», Social Science \& Medicine, vol. 48, pp. 745-755.

BONDAS, Terese \& HALL, Elisabeth O.C. (2007): «Challenges in approaching metasynthesis research», Qualitative Health Research, vol. 17, nํ1, pp. 113-121.

BURKE, Mary Anne \& EICHLER, Margrit (2006): The BIAS FREE Framework: a practical tool for identifying and eliminating social biases in health research. Global Forum for Health Research. Disponible en:

ht tp://www.globalforumhealth.org/Site/ 002 _What $\% 20$ we $\% 20 \mathrm{~d}$ o/005_Publications/010__BIAS\%20FREE.php

BOURDIEU, Pierre (1995): «Una duda radical», en Bourdieu, Pierre \& Wacquant, Loic (1995). Respuestas. Por una antropología reflexiva, México, Grijalbo, pp. 177-184.

BOURDIEU, Pierre (2002): El oficio de sociológo, Madrid, Siglo XXI.

CERÓN-MIRELES, P.; SÁNCHEZ-CARRILLO, C. I.; ROBLEDO-VERA, C. et al. (2006): «Aplicación de la perspectiva de género en artículos publicados en cuatro revistas nacionales de salud, México, 2000-2003», Salud Pública de México, vol. 48, no 4, pp. 332-340.

COMELLES, Josep María (2004): «El regreso de las culturas. Diversidad cultural y práctica médica en el siglo XXI», en G. Fernández Juárez (coord.) Salud e Interculturalidad en América Latina. Perspectivas antropológicas, Quito, Ediciones Abya-Yala, pp. 17-30.

COMELLES, Josep María \& BERNAL, Mariola (2007): «El hecho migratorio y la vulnerabilidad del sistema sanitario en España», Humanitas Humanidades Médicas, 13, pp. 1-17.

EICHLER, Magrit (2001): «Moving forward: Measuring gender bias and more», en: VV.AA. Gender Based Analysis in Public Health Research Policy and Practice. Documentation of the International Workshop in Berlin, 2001. Disponible en:

http://www.ifg-gs.tu-berlin.de/workshop/workshop-doku.pdf

GARCÍA CASTAÑO, Francisco J, GRANADOS MARTÍNEZ A. \& PULIDO MOYANO R.A. (1999): «Reflexiones en diversos ámbitos de construcción de la diferencia», en F.J. García Castaño y A. Granados Martínez (eds.) Lecturas para educación intercultural, Madrid, Trotta, pp. 39-59.

GIJÓN, María Teresa, JIMÉNEZ RODRIGO, María Luisa \& MARTÍNEZ MORANTE, Emilia. (2006): «Más allá de la diferencia, tras el cristal de la diversidad. La «inmigración» en la literatura biomédica en España», en G. Fernández Juáres (coord): $\mathrm{Sa}$ lud e Interculturalidad en América Latina. Antropología de la Salud y Crítica Intercultural, Quito, Ediciones ABYA YALA, pp. 115-132.

GÓMEZ RODRÍGUEZ, Amparo (2004): La estirpe maldita. La construcción científica de lo femenino, Madrid, Minerva. 
HARAWAY, Donna (1988): «Situated knowledges. The science question in feminism and the privilege of partial perspective», Feminist Studies, vol. 14, nº 3, pp. 575-599.

HARAWAY, Donna (1999): «Las promesas de los monstruos: Una política regeneradora para otros inapropiados/ables», Política y Sociedad, vol. 30, pp. 121-163.

HARDING, Sandra (1991): Whose Science? Whose Knowledge? Thinking from women's lives, Buckingham, Open University Press.

HARDING, Sandra (1996): Ciencia y feminismo, Madrid, Morata.

HARDING, Sandra (2003): «Representing reality: the critical realism project», Feminist Economics, vol. 9, no 1, pp. 151-159.

IM, Eun-Ok (2000): «A feminist critique of research on women's work and health», Health Care for Women International, vol. 21, pp.105-109.

INHORN, Marcia \& WHITTLE, Lisa (2001): «Feminism meets the "new" epidemiologies: Toward an appraisal of antifeminist biases in epidemiological research on women's health», Social Science \& Medicine, vol. 53, pp. 553-67.

IRANZO, Juan Manuel \& BLANCO, José Rubén (1999): Sociología del conocimiento científico, Madrid, Centro de Investigaciones Sociológicas.

KELLER, Evelyn Fox (1982): «Feminism and science», Signs, vol. 7, pp. 589-602.

JIMÉNEZ RODRIGO, María Luisa, MARTÍNEZ MORANTE, Emilia \& GARCIA CALVENTE, María del Mar (2008): «Propuesta de una herramienta para la evaluación de género de las políticas de investigación en salud», en C. Miqueo, M.J. Barral y C. Magallón (coord.): Estudios iberoamericanos en ciencia, tecnología y salud: GENCIBER, Zaragoza, Prensas Universitarias de Zaragoza, pp. 129-134.

KAPLAN, J. \& BENNET, T. (2003): «Use of the race and ethnicity in biomedical publication», Journal of American Medical Association, vol. 289, nº 20, pp. 2709-16.

LATOUR, Bruno (1992): Ciencia en acción: cómo seguir a los científicos e ingenieros a través de la sociedad, Barcelona, Labor.

MAQUEIRA, Virginia (2005): «Género, diferencia y desigualdad», en E. Beltrán. \& V. Maqueira (eds.). Feminismos. Debates contemporáneos, Madrid, Alianza, pp. 127190.

McCALL, Leslie (2005): «The complexity of intersectionality», Signs, vol. 30, n 3, pp. 1771-1800.

MENÉNDEZ, Eduardo (1990): Morir de Alcohol. Saber y hegemonía médica, México, Editorial Alianza.

MENÉNDEZ, Eduardo (2003): «Modelo de atención de los padecimientos: de exclusiones teóricas y articulaciones prácticas», Ciencia y Saúde Colectiva, vol. 8, no 1 , pp. 185-207.

MENÉNDEZ, Eduardo \& DI PARDO, Renée (1996): De algunos alcoholismos y algunos saberes. Atención primaria y proceso de alcoholización, México, CIESAS. Col. M. Othon de Mendizabal.

MIQUEO, Consuelo (2001): «Semiología del androcentrismo. Teorías sobre reproducción de Andrés Piquer y François Broussais», en: C. Miqueo, C. Tomás; C. Tejero et al. Perspectivas de género en salud. Fundamentos científicos y socioprofesionales de diferencias sexuales no previstas, Madrid, Minerva Ediciones, pp. 97-134.

MOORE, Henrietta (1999): Antropología y feminismo, Madrid, Cátedra.

MOZO, Carmen \& TENA, Fernando (2003): Antropología de los géneros en Andalucía. De viajeros, antropólogos y sexualidad, Sevilla, Mergablum. 
NASH, Mary (2001): «Diversidad, multiculturalismos e identidades: perspectivas de género», en M. Nash \& D. Marre (ed.) Multiculturalismos y género. Un estudio interdisciplinar, Barcelona, Edicions Bellaterra, pp. 21-47.

NENCEL, Lorraine \& PELS, Meter (1991): Constructing knowledge: authority and critique in social science, London,

NOBLIT, George W. \& HARE, R. Dwight (1988): Meta-ethnography: synthesizing qualitative studies, Newbury Park CA, Sage.

OAKLEY, Ann (1988): «Science, gender and women's liberation: an argument against postmodernism», Women's Studies International Forum, vol 21, no 2, pp. 133-146.

ORGANIZACIÓN INTERNACIONAL PARA LAS MIGRACIONES (OIM) (2006): Glosario sobre migraciones. Ginebra, OIT.

ORTIZ GÓMEZ, Teresa (2002): «El papel del género en la construcción histórica del conocimiento científico sobre la mujer», en E. Ramos (ed.) La salud de las mujeres: hacia la igualdad de género en salud, Madrid, Ministerio de Trabajo y Asuntos Sociales / Instituto de la Mujer, pp. 29-49.

ORTIZ GÓMEZ, Teresa (2006): Medicina, historia y género. 130 años de investigación feminista, Oviedo, KRK.

RITZER, George (1991): «Metatheorizing in sociology», Sociological Forum, vol.5, pp.3-15.

RODRÍGUEZ, Vicente; CASADO, María Ángeles \& HUBER, Andreas (eds.) (2005): La migración de europeos retirados en España, Madrid, Consejo Superior de Investigaciones Científicas.

ROSSER, Sue (1994): «Gender bias in clinical research: the difference it makes», en A. Dan (ed.) Reforming women's health: multidisciplinary research and practice, London, Sage Publication, pp. 25-30.

SENIOR, P.A. \& BHOPAL, R. (1994): «Ethnicity as a variable in epidemiological research», British Medical Journal, vol. 309, pp. 327-30.

SOLÉ, Carlota (dir.) (2006): Inmigración comunitaria, ¿discriminación inversa?, Madrid, Anthropos.

THORNE, Sally; JENSEN, Louise; KEARNEY, Margaret et al (2004): «Qualitative Metasynthesis: Reflections on methodological orientation and ideological agenda», Qualitative Health Research, 1342-1365.

TUBERT, Silvia (2003): Del sexo al género. Los equívocos de un concepto, Madrid, Cátedra.

ZHAO, Shanyang (1991): «Metatheory, metamethod, meta-data-analysis: What, why, and how?», Sociological Perspectives, vol. 34, no 3, pp. 377-390.

ZIMMER, Lela (2006): «Qualitative meta-synthesis: a question of dialoguing with texts», Journal of Advanced Nursing, vol. 53, nº 3, pp. 311-318.

\section{RESUMEN}

En este trabajo proponemos la práctica de la metainvestigación como herramienta de revisión y análisis de las publicaciones científicas. Este concepto hace referencia a la necesidad de reflexionar y evaluar críticamente qué y cómo se investiga como estrategia fundamental de avance científico. Hemos centrado nuestro interés en el campo de la biomedicina y en su estudio del proceso de sa- 
lud-enfermedad-atención en inmigrantes de origen extranjero. Nuestro objetivo es analizar cómo son seleccionadas, construidas y abordadas las cuestiones científicamente relevantes sobre esta población, y específicamente, cómo afectan los sesgos derivados del etnocentrismo/etnicismo y del androcentrismo/sexismo a este proceso y a sus resultados. Para ello, analizamos desde la diversidad cultural y el género los artículos científicos sobre inmigración publicados desde 1984 a 2006 indexados en el Índice Médico Español-CSIC. El modo en qué son definidas las temáticas sanitaria y académicamente significativas responde, en gran parte de los artículos, a visiones etnocéntricas y androcéntricas que, además de reforzar estereotipos y desigualdades sociales en salud, implica limitaciones en la validez y rigor científicos, con las consecuencias que esto conlleva en su transferencia a la práctica sanitaria.

\title{
PALABRAS CLAVE
}

Producción científica, género, diversidad cultural, inmigración, salud.

\begin{abstract}
In this paper, metaresearch is proposed as a tool for review and analysis of scientific publications. This concept refers to the need of critically evaluate and reflect what and how people research as a fundamental strategy of scientific progress. Our interest is situated in the field of the biomedicine and the study of health-illness-care process in foreign immigrants. The objective is to analyse how scientifically relevant questions about this population are selected, constructed and broached. Specifically, we aim to evaluate how ethnocentric/ethnicist and andocentric/sexist biases affect this process and its results. We analyse from a cultural diversity and gender perspective the scientific articles on immigration published from 1984 to 2006 indexed in the Spanish Medical Index-CSIC. In the majority of the articles, the definition of the sanitary and academic significant questions responds to ethnocentric and androcentric visions that strengthen social stereotypes and inequalities. Furthermore, they involve limitations of scientific rigour and validity, with consequences in the sanitary practice.
\end{abstract}

\section{KEYWORDS}

Scientific production, gender, cultural diversity, immigration, health. 